\section{ㄷ. REPORTS}

ISSN 1600-5368

\section{Crystal structure of poly $\left[\left\{\mu-N, N^{\prime}\right.\right.$-bis- [(pyridin-4-yl)methyl]oxalamide]- $\mu$ - oxalato-cobalt(II)]}

\section{Hengye Zou and Yanjuan Qi*}

Department of Chemistry, Changchun Normal University, Changchun 130032, People's Republic of China. *Correspondence e-mail: qiyjchem@163.com

Received 26 June 2014; accepted 3 July 2014

Edited by M. Bolte, Goethe-Universität Frankfurt, Germany

In the polymeric title compound, $\left[\mathrm{Co}\left(\mathrm{C}_{2} \mathrm{O}_{4}\right)\left(\mathrm{C}_{14} \mathrm{H}_{14} \mathrm{~N}_{4} \mathrm{O}_{2}\right)\right]_{n}$, the $\mathrm{Co}^{\mathrm{II}}$ atom is six-coordinated by two $\mathrm{N}$ atoms from symmetry-related bis[(pyridin-4-yl)methyl]oxalamide (BPMO) ligands and four $\mathrm{O}$ atoms from two centrosymmetric oxalate anions in a distorted octahedral coordination geometry. The $\mathrm{Co}^{\mathrm{II}}$ atoms are linked by the oxalate anions into a chain running parallel to [100]. The chains are linked by the BPMO ligands into a three-dimensional architecture. In addition, $\mathrm{N}-\mathrm{H} \cdots \mathrm{O}$ hydrogen bonds stabilize the crystal packing.

Keywords: crystal structure; metal-organic framework; cobalt(II); oxalate anion; hydrogen bonds.

CCDC reference: 1012047

\section{Related literature}

For information on compounds with metal-organic framework structures, see: Kitagawa et al. (2004); Ma et al. (2009); Li et al. (2005); Wang et al. (2007). For related $\mathrm{Co}^{\mathrm{II}}$ complexes, see: Ma et al. (2005).

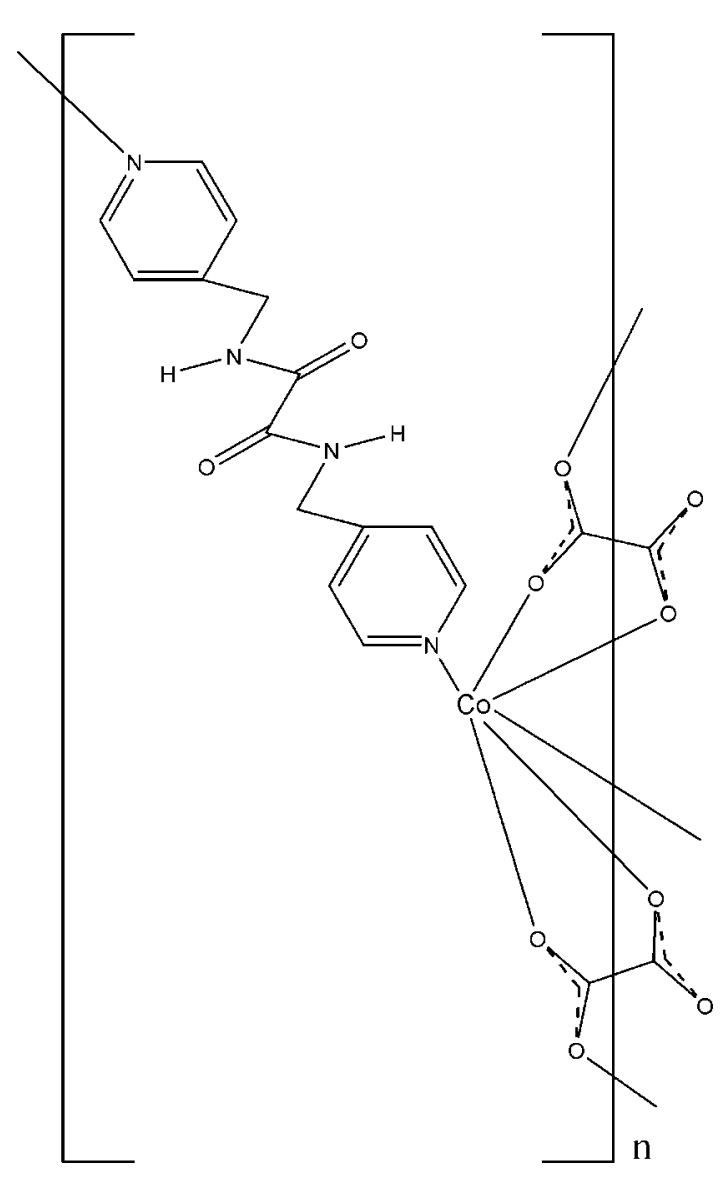

2. Experimental

2.1. Crystal data

$\left[\mathrm{Co}\left(\mathrm{C}_{2} \mathrm{O}_{4}\right)\left(\mathrm{C}_{14} \mathrm{H}_{14} \mathrm{~N}_{4} \mathrm{O}_{2}\right)\right]$ $M_{r}=417.24$

Monoclinic, $P 2_{1} / c$

$a=8.4143(12) \AA$

$b=24.421(4) \AA$

$c=9.2884(14) \AA$

$\beta=113.322(2)^{\circ}$

$V=1752.7(4) \AA^{3}$

$Z=4$

Mo $K \alpha$ radiation

$\mu=1.02 \mathrm{~mm}^{-1}$

$T=293 \mathrm{~K}$

$0.43 \times 0.25 \times 0.25 \mathrm{~mm}$

\subsection{Data collection}

Bruker SMART APEXII CCD diffractometer

Absorption correction: multi-scan

(SADABS; Sheldrick, 1996)

$T_{\min }=0.740, T_{\max }=0.785$

\subsection{Refinement}

$$
\begin{aligned}
& R\left[F^{2}>2 \sigma\left(F^{2}\right)\right]=0.065 \\
& w R\left(F^{2}\right)=0.149 \\
& S=0.98 \\
& 4254 \text { reflections }
\end{aligned}
$$

11121 measured reflections 4254 independent reflections 2027 reflections with $I>2 \sigma(I)$ $R_{\text {int }}=0.085$
244 parameters

$\mathrm{H}$-atom parameters constrained $\Delta \rho_{\max }=0.49 \mathrm{e} \AA^{-3}$

$\Delta \rho_{\min }=-0.39 \mathrm{e}^{-3}$ 
Table 1

Hydrogen-bond geometry $\left(\AA,^{\circ}\right)$.

\begin{tabular}{lllll}
\hline$D-\mathrm{H} \cdots A$ & $D-\mathrm{H}$ & $\mathrm{H} \cdots A$ & $D \cdots A$ & $D-\mathrm{H} \cdots A$ \\
\hline $\mathrm{N} 3-\mathrm{H} 3 \cdots \mathrm{O}^{\mathrm{i}}$ & 0.86 & 2.14 & $2.863(5)$ & 142 \\
\hline
\end{tabular}

Symmetry code: (i) $x,-y+\frac{1}{2}, z+\frac{1}{2}$.

Data collection: APEX2 (Bruker, 2002); cell refinement: SAINT (Bruker, 2002); data reduction: $S A I N T$; $\operatorname{program}(\mathrm{s})$ used to solve structure: SHELXTL (Sheldrick, 2008); program(s) used to refine structure: SHELXTL; molecular graphics: Mercury (Macrae et al., 2006); software used to prepare material for publication: SHELXTL and publCIF (Westrip, 2010).

\section{Acknowledgements}

We thank the Science Foundation of Jilin Province (No. 20140101121JC).
Supporting information for this paper is available from the IUCr electronic archives (Reference: BT6986).

\section{References}

Bruker (2002). APEX2 and SAINT. Bruker AXS Inc., Madison, Wisconsin, USA.

Kitagawa, S., Kitaura, R. \& Noro, S. (2004). Angew. Chem. Int. Ed. 43, 2334 2375.

Li, F. F., Ma, J. F., Song, S. Y., Yang, J., Liu, Y. Y. \& Su, Z. M. (2005). Inorg. Chem. 44, 9374-9383.

Ma, L. F., Wang, Y. Y., Liu, J. Q., Yang, G. P., Du, M. \& Wang, L. Y. (2009). Eur. J. Inorg. Chem. pp. 147-254.

Ma, J. F., Yang, J., Li, S. L. \& Song, S. Y. (2005). Cryst. Growth Des. 5, 807-812. Macrae, C. F., Edgington, P. R., McCabe, P., Pidcock, E., Shields, G. P., Taylor, R., Towler, M. \& van de Streek, J. (2006). J. Appl. Cryst. 39, 453-457. Sheldrick, G. M. (1996). SADABS. University of Göttingen, Germany.

Sheldrick, G. M. (2008). Acta Cryst. A64, 112-122.

Wang, G.-H., Li, Z.-G., Xu, J.-W. \& Hu, N.-H. (2007). Acta Cryst. E63, m289$\mathrm{m} 291$.

Westrip, S. P. (2010). J. Appl. Cryst. 43, 920-925. 


\section{supporting information}

Acta Cryst. (2014). E70, m307-m308 [doi:10.1107/S1600536814015608]

\section{Crystal structure of poly[ $\left\{\mu-N, N^{\prime}-\right.$ bis $[($ pyridin-4-yl)methyl $]$ oxalamide $\}-\mu$-oxalato- cobalt(II)]}

\section{Hengye Zou and Yanjuan Qi}

\section{S1. Comment}

Design of effective ligands and the proper choice of metal centers are the keys to design and construct novel metalorganic frameworks (Kitagawa et al., 2004; Ma et al., 2009). These complexes can be specially designed by the careful selection of metal cations with preferred coordination geometries, the nature of the anions, the structure of the connecting ligands, and the reaction conditions (Li et al., 2005; Wang et al., 2007). We selected oxalic acid as an organic carboxylate anion and $N, N^{\prime}$-Bis-pyridin-4-ylmethyl-oxalamide (BPMO) as a $\mathrm{N}$-donor neutral ligand, generating a coordination compound, $\left[\mathrm{Co}\left(\mathrm{C}_{2} \mathrm{O}_{4}\right)(\mathrm{BPMO})\right]_{\mathrm{n}}$, which is reported here.

In the asymmetric unit of the title compound, $\left[\mathrm{Co}\left(\mathrm{C}_{2} \mathrm{O}_{4}\right)(\mathrm{BPMO})\right]_{\mathrm{n}}$, the central $\mathrm{Co}^{\mathrm{II}}$ is six-coordinated by two nitrogen atoms from different BPMO ligands and four oxygen atoms from two oxalate anions in a distorted octahedral coordination geometry. The $\mathrm{Co}-\mathrm{N}$ and $\mathrm{Co}-\mathrm{O}$ distances are comparable to those found in other crystallographically characterized $\mathrm{Co}^{\mathrm{II}}$ complexes (Ma et al., 2005). The $\mathrm{Co}^{\mathrm{II}}$ atoms are linked by the oxalate anions to give a one-dimensional chain. The chains are linked by BPMO ligands and extend the chains into a three-dimensional supramolecular architecture. Moreover, the hydrogen bonds between the N-donor neutral ligand and oxalate, are crucial for stabilizing the three-dimensional framework.

\section{S2. Experimental}

The synthesis was performed under hydrothermal conditions. A mixture of $\mathrm{Co}\left(\mathrm{CH}_{3} \mathrm{COO}\right)_{2} \cdot 4\left(\mathrm{H}_{2} \mathrm{O}\right),(0.2 \mathrm{mmol}, 0.05 \mathrm{~g})$, $N, N^{\prime}$-Bis-pyridin-4-ylmethyl-oxalamide $(0.2 \mathrm{mmol}, 0.054 \mathrm{~g})$, sodium oxalate $(0.2 \mathrm{mmol}, 0.026 \mathrm{~g})$ and $\mathrm{H}_{2} \mathrm{O}(15 \mathrm{ml})$ in a 25 $\mathrm{ml}$ stainless steel reactor with a Teflon liner was heated from 293 to $443 \mathrm{~K}$ in $2 \mathrm{~h}$ and a constant temperature was maintained at $443 \mathrm{~K}$ for $72 \mathrm{~h}$, after which the mixture was cooled to $298 \mathrm{~K}$. Pink crystals of (I) were recovered from the reaction.

\section{S3. Refinement}

All $\mathrm{H}$ atoms on $\mathrm{C}$ and $\mathrm{N}$ atoms atoms were poisitioned geometrically and refined as riding atoms with $U_{\text {iso }}(\mathrm{H})=1.2$ $U_{\text {eq }}(\mathrm{C}, \mathrm{N})$. 


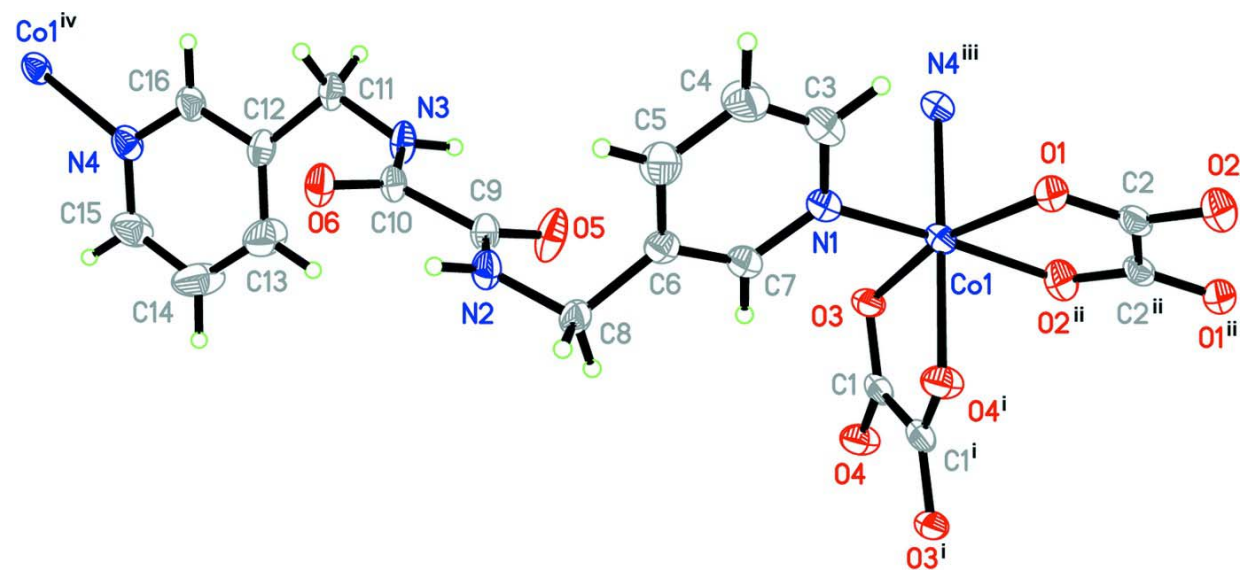

\section{Figure 1}

A view of the molecule of (I). Displacement ellipsoids are drawn at the $30 \%$ probability level. (i) $-x+1,-y,-z+1$; (ii) $x,-y,-z+1$; (iii) $x,-y+1 / 2, z+1 / 2$; (iv) $x+1,-y+1 / 2, z-1 / 2$.

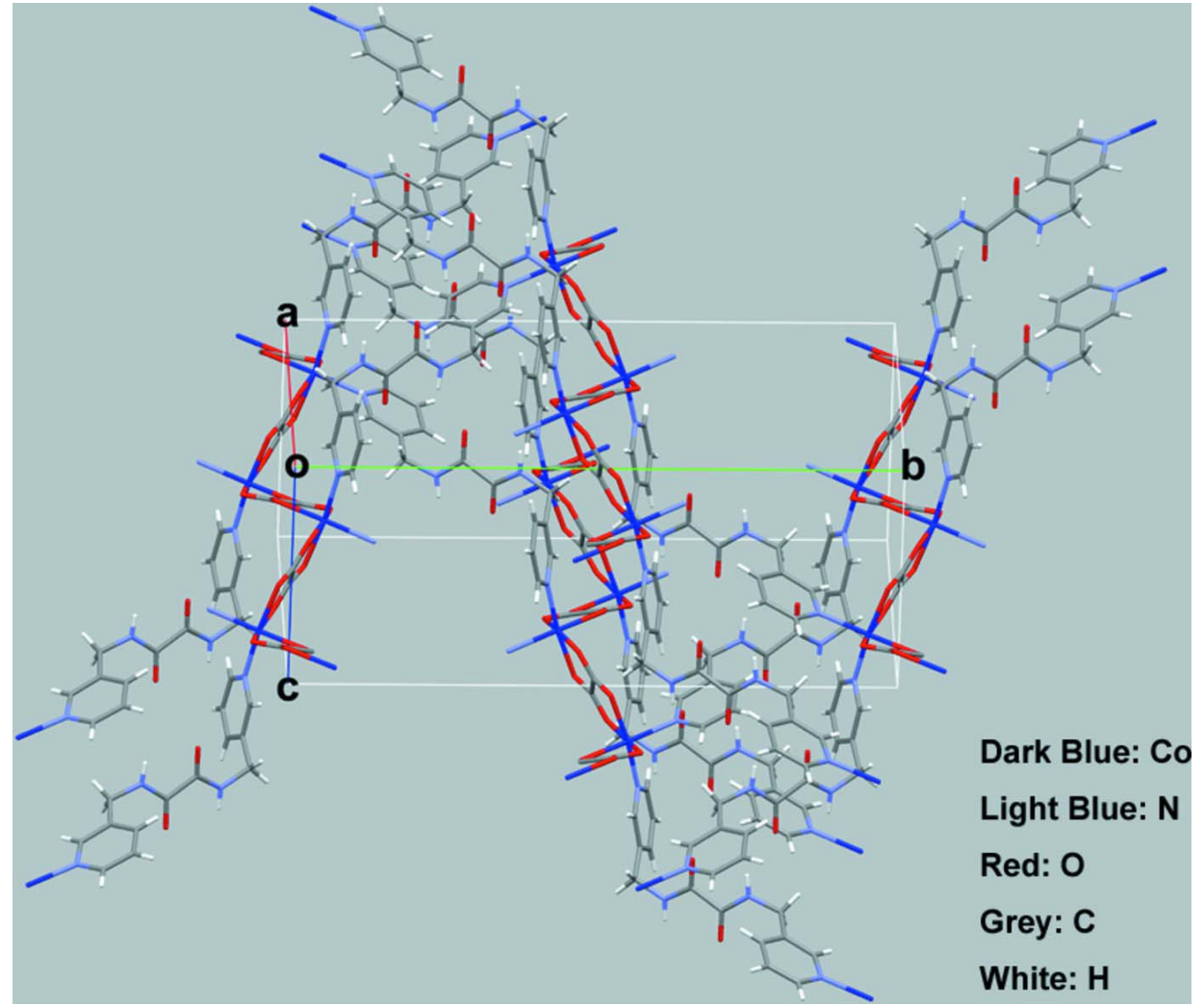

\section{Figure 2}

View of the three-dimensional structure of (I).

\section{Poly[ $\left\{\mu-N, N^{\prime}-\right.$ bis[(pyridin-4-yl)methyl]oxalamide\}- $\mu$-oxalato-cobalt(II)]}

\section{Crystal data}

$\left[\mathrm{Co}\left(\mathrm{C}_{2} \mathrm{O}_{4}\right)\left(\mathrm{C}_{14} \mathrm{H}_{14} \mathrm{~N}_{4} \mathrm{O}_{2}\right)\right]$

$M_{r}=417.24$

Monoclinic, $P 2{ }_{1} / c$
Hall symbol: -P 2ybc

$a=8.4143$ (12) $\AA$

$b=24.421(4) \AA$ 
$c=9.2884(14) \AA$

$\beta=113.322(2)^{\circ}$

$V=1752.7(4) \AA^{3}$

$Z=4$

$F(000)=852$

$D_{\mathrm{x}}=1.581 \mathrm{Mg} \mathrm{m}^{-3}$

Mo $K \alpha$ radiation, $\lambda=0.71073 \AA$

Data collection

Bruker SMART APEXII CCD

diffractometer

Radiation source: fine-focus sealed tube

Graphite monochromator

phi and $\omega$ scans

Absorption correction: multi-scan

(SADABS; Sheldrick, 1996)

$T_{\min }=0.740, T_{\max }=0.785$

Refinement

Refinement on $F^{2}$

Least-squares matrix: full

$R\left[F^{2}>2 \sigma\left(F^{2}\right)\right]=0.065$

$w R\left(F^{2}\right)=0.149$

$S=0.98$

4254 reflections

244 parameters

0 restraints

Primary atom site location: structure-invariant

direct methods
Cell parameters from 4380 reflections

$\theta=1.7-22.8^{\circ}$

$\mu=1.02 \mathrm{~mm}^{-1}$

$T=293 \mathrm{~K}$

Block, pink

$0.43 \times 0.25 \times 0.25 \mathrm{~mm}$

11121 measured reflections

4254 independent reflections

2027 reflections with $I>2 \sigma(I)$

$R_{\text {int }}=0.085$

$\theta_{\max }=28.4^{\circ}, \theta_{\min }=2.5^{\circ}$

$h=-11 \rightarrow 10$

$k=-32 \rightarrow 32$

$l=-12 \rightarrow 10$

Secondary atom site location: difference Fourier map

Hydrogen site location: inferred from

neighbouring sites

$\mathrm{H}$-atom parameters constrained

$w=1 /\left[\sigma^{2}\left(F_{\mathrm{o}}^{2}\right)+(0.0591 P)^{2}\right]$

where $P=\left(F_{\mathrm{o}}^{2}+2 F_{\mathrm{c}}^{2}\right) / 3$

$(\Delta / \sigma)_{\max }<0.001$

$\Delta \rho_{\max }=0.49$ e $\AA^{-3}$

$\Delta \rho_{\min }=-0.39$ e $\AA^{-3}$

Special details

Geometry. All e.s.d.'s (except the e.s.d. in the dihedral angle between two 1.s. planes) are estimated using the full covariance matrix. The cell e.s.d.'s are taken into account individually in the estimation of e.s.d.'s in distances, angles and torsion angles; correlations between e.s.d.'s in cell parameters are only used when they are defined by crystal symmetry. An approximate (isotropic) treatment of cell e.s.d.'s is used for estimating e.s.d.'s involving 1.s. planes.

Refinement. Refinement of $F^{2}$ against ALL reflections. The weighted $R$-factor $w R$ and goodness of fit $S$ are based on $F^{2}$, conventional $R$-factors $R$ are based on $F$, with $F$ set to zero for negative $F^{2}$. The threshold expression of $F^{2}>\sigma\left(F^{2}\right)$ is used only for calculating $R$-factors(gt) etc. and is not relevant to the choice of reflections for refinement. $R$-factors based on $F^{2}$ are statistically about twice as large as those based on $F$, and $R$ - factors based on ALL data will be even larger.

Fractional atomic coordinates and isotropic or equivalent isotropic displacement parameters $\left(\AA^{2}\right)$

\begin{tabular}{lllll}
\hline & $x$ & $y$ & $z$ & $U_{\text {iso }} / U_{\text {eq }}$ \\
\hline C1 & $0.5475(6)$ & $0.02677(18)$ & $0.5426(6)$ & $0.0391(12)$ \\
C2 & $-0.0727(6)$ & $0.00051(18)$ & $0.4182(6)$ & $0.0373(11)$ \\
C3 & $0.0325(7)$ & $0.0938(2)$ & $0.0435(7)$ & $0.0588(15)$ \\
H3A & -0.0628 & 0.0983 & 0.0692 & $0.071^{*}$ \\
C4 & $0.0141(7)$ & $0.1061(2)$ & $-0.1055(7)$ & $0.0644(16)$ \\
H4 & -0.0906 & 0.1187 & -0.1797 & $0.077^{*}$ \\
C5 & $0.1578(7)$ & $0.0990(2)$ & $-0.1424(6)$ & $0.0553(15)$ \\
H5 & 0.1507 & 0.1079 & -0.2422 & $0.066^{*}$ \\
C6 & $0.3094(6)$ & $0.07904(17)$ & $-0.0329(6)$ & $0.0358(11)$ \\
C7 & $0.3141(6)$ & $0.06856(18)$ & $0.1129(6)$ & $0.0413(12)$
\end{tabular}




$\begin{array}{lllll}\text { H7 } & 0.4172 & 0.0555 & 0.1884 & 0.050^{*} \\ \text { C8 } & 0.4646(6) & 0.06836(16) & -0.0724(6) & 0.0398(12) \\ \text { H8A } & 0.5578 & 0.0537 & 0.0195 & 0.048^{*} \\ \text { H8B } & 0.4343 & 0.0409 & -0.1543 & 0.048^{*} \\ \text { C9 } & 0.5876(6) & 0.15839(19) & -0.0278(6) & 0.0372(11) \\ \text { C10 } & 0.6436(5) & 0.20856(18) & -0.0958(6) & 0.0353(11) \\ \text { C11 } & 0.7359(6) & 0.30333(18) & -0.0376(6) & 0.0411(12) \\ \text { H11A } & 0.7224 & 0.3306 & 0.0328 & 0.049^{*} \\ \text { H11B } & 0.6576 & 0.3130 & -0.1432 & 0.049^{*} \\ \text { C12 } & 0.9189(6) & 0.30606(19) & -0.0279(5) & 0.0396(12) \\ \text { C13 } & 1.0379(7) & 0.2660(2) & 0.0275(7) & 0.0700(18) \\ \text { H13 } & 1.0094 & 0.2334 & 0.0632 & 0.084^{*} \\ \text { C14 } & 1.2033(7) & 0.2734(2) & 0.0312(8) & 0.083(2) \\ \text { H14 } & 1.2859 & 0.2458 & 0.0666 & 0.100^{*} \\ \text { C15 } & 1.2411(7) & 0.3229(2) & -0.0193(7) & 0.0631(16) \\ \text { H15 } & 1.3517 & 0.3281 & -0.0165 & 0.076^{*} \\ \text { C16 } & 0.9683(6) & 0.35427(19) & -0.0767(6) & 0.0431(12) \\ \text { H16 } & 0.8861 & 0.3819 & -0.1153 & 0.052^{*} \\ \text { N1 } & 0.1806(5) & 0.07571(16) & 0.1551(5) & 0.0455(11) \\ \text { N2 } & 0.5257(5) & 0.11716(15) & -0.1243(4) & 0.0403(10) \\ \text { H2 } & 0.5213 & 0.1190 & -0.2183 & 0.048^{*} \\ \text { N3 } & 0.6855(4) & 0.25082(15) & 0.0010(4) & 0.0423(10) \\ \text { H3 } & 0.6826 & 0.2467 & 0.0918 & 0.051^{*} \\ \text { N4 } & 1.1276(5) & 0.36374(16) & -0.0717(5) & 0.0439(10) \\ \text { O1 } & -0.0491(4) & 0.02611(12) & 0.3106(4) & 0.0434(8) \\ \text { O2 } & -0.2077(4) & -0.02540(13) & 0.4033(4) & 0.0503(9) \\ \text { O3 } & 0.4618(4) & 0.07115(12) & 0.5055(4) & 0.0446(9) \\ \text { O4 } & 0.6991(4) & 0.02217(12) & 0.6401(4) & 0.0494(9) \\ \text { O5 } & 0.5999(5) & 0.15868(13) & 0.1070(4) & 0.0612(11) \\ \text { O6 } & 0.6455(4) & 0.20801(12) & -0.2267(4) & 0.0464(8) \\ \text { Co1 } & 0.20072(8) & 0.05586(2) & 0.38091(8) & 0.0405(2)\end{array}$

Atomic displacement parameters $\left(\AA^{2}\right)$

\begin{tabular}{lllllll}
\hline & $U^{11}$ & $U^{22}$ & $U^{33}$ & $U^{12}$ & $U^{13}$ & $U^{23}$ \\
\hline C1 & $0.035(3)$ & $0.043(3)$ & $0.049(3)$ & $0.003(2)$ & $0.026(3)$ & $-0.001(2)$ \\
C2 & $0.034(3)$ & $0.033(2)$ & $0.051(3)$ & $0.002(2)$ & $0.023(2)$ & $-0.008(2)$ \\
C3 & $0.050(4)$ & $0.067(4)$ & $0.068(4)$ & $0.013(3)$ & $0.034(3)$ & $0.009(3)$ \\
C4 & $0.043(4)$ & $0.087(4)$ & $0.053(4)$ & $0.018(3)$ & $0.009(3)$ & $0.015(3)$ \\
C5 & $0.063(4)$ & $0.061(4)$ & $0.044(4)$ & $0.003(3)$ & $0.023(3)$ & $0.001(3)$ \\
C6 & $0.037(3)$ & $0.026(2)$ & $0.043(3)$ & $-0.004(2)$ & $0.015(3)$ & $-0.005(2)$ \\
C7 & $0.034(3)$ & $0.044(3)$ & $0.046(3)$ & $0.006(2)$ & $0.016(2)$ & $0.004(3)$ \\
C8 & $0.053(3)$ & $0.027(2)$ & $0.048(3)$ & $-0.004(2)$ & $0.029(3)$ & $-0.001(2)$ \\
C9 & $0.035(3)$ & $0.041(3)$ & $0.037(3)$ & $-0.010(2)$ & $0.016(2)$ & $-0.002(2)$ \\
C10 & $0.034(3)$ & $0.039(3)$ & $0.038(3)$ & $-0.010(2)$ & $0.019(2)$ & $-0.001(2)$ \\
C11 & $0.048(3)$ & $0.040(3)$ & $0.044(3)$ & $-0.010(2)$ & $0.027(3)$ & $0.001(2)$ \\
C12 & $0.043(3)$ & $0.042(3)$ & $0.034(3)$ & $-0.013(2)$ & $0.016(2)$ & $-0.001(2)$ \\
C13 & $0.052(4)$ & $0.045(3)$ & $0.109(6)$ & $-0.009(3)$ & $0.028(4)$ & $0.015(3)$
\end{tabular}


supporting information

\begin{tabular}{lllllll}
$\mathrm{C} 14$ & $0.050(4)$ & $0.046(4)$ & $0.142(7)$ & $0.003(3)$ & $0.026(4)$ & $0.016(4)$ \\
$\mathrm{C} 15$ & $0.045(3)$ & $0.048(3)$ & $0.104(5)$ & $-0.003(3)$ & $0.036(3)$ & $-0.003(3)$ \\
$\mathrm{C} 16$ & $0.039(3)$ & $0.045(3)$ & $0.052(3)$ & $-0.006(2)$ & $0.024(3)$ & $0.001(3)$ \\
$\mathrm{N} 1$ & $0.041(3)$ & $0.047(2)$ & $0.054(3)$ & $0.0103(19)$ & $0.024(2)$ & $0.009(2)$ \\
$\mathrm{N} 2$ & $0.052(3)$ & $0.041(2)$ & $0.035(2)$ & $-0.0080(19)$ & $0.026(2)$ & $-0.0039(19)$ \\
$\mathrm{N} 3$ & $0.052(3)$ & $0.045(2)$ & $0.038(3)$ & $-0.0204(19)$ & $0.026(2)$ & $-0.005(2)$ \\
$\mathrm{N} 4$ & $0.039(2)$ & $0.042(2)$ & $0.058(3)$ & $-0.0088(19)$ & $0.027(2)$ & $-0.005(2)$ \\
O1 & $0.040(2)$ & $0.044(2)$ & $0.048(2)$ & $0.0012(15)$ & $0.0202(17)$ & $0.0061(17)$ \\
O2 & $0.042(2)$ & $0.057(2)$ & $0.055(2)$ & $-0.0090(17)$ & $0.0222(18)$ & $-0.0050(18)$ \\
O3 & $0.0356(19)$ & $0.0339(18)$ & $0.070(3)$ & $0.0063(14)$ & $0.0269(18)$ & $0.0041(16)$ \\
O4 & $0.037(2)$ & $0.041(2)$ & $0.065(3)$ & $0.0040(16)$ & $0.0145(19)$ & $-0.0052(17)$ \\
O5 & $0.098(3)$ & $0.050(2)$ & $0.044(2)$ & $-0.036(2)$ & $0.037(2)$ & $-0.0086(18)$ \\
O6 & $0.064(2)$ & $0.044(2)$ & $0.041(2)$ & $-0.0107(16)$ & $0.0318(19)$ & $-0.0026(16)$ \\
Co1 & $0.0349(4)$ & $0.0385(4)$ & $0.0551(5)$ & $0.0057(3)$ & $0.0253(3)$ & $0.0030(3)$ \\
& & & & & & \\
\hline
\end{tabular}

Geometric parameters $\left(\AA,{ }^{\circ}\right)$

\begin{tabular}{|c|c|c|c|}
\hline $\mathrm{C} 1-\mathrm{O} 4$ & $1.243(5)$ & $\mathrm{C} 11-\mathrm{N} 3$ & $1.440(5)$ \\
\hline $\mathrm{C} 1-\mathrm{O} 3$ & $1.271(5)$ & $\mathrm{C} 11-\mathrm{C} 12$ & $1.508(6)$ \\
\hline $\mathrm{C} 1-\mathrm{C}^{\mathrm{i}}$ & $1.573(9)$ & $\mathrm{C} 11-\mathrm{H} 11 \mathrm{~A}$ & 0.9700 \\
\hline $\mathrm{C} 2-\mathrm{O} 2$ & $1.259(5)$ & $\mathrm{C} 11-\mathrm{H} 11 \mathrm{~B}$ & 0.9700 \\
\hline $\mathrm{C} 2-\mathrm{O} 1$ & $1.260(5)$ & $\mathrm{C} 12-\mathrm{C} 13$ & $1.348(6)$ \\
\hline $\mathrm{C} 2-\mathrm{C} 2^{\mathrm{ii}}$ & $1.527(9)$ & $\mathrm{C} 12-\mathrm{C} 16$ & $1.383(6)$ \\
\hline $\mathrm{C} 3-\mathrm{N} 1$ & $1.342(6)$ & $\mathrm{C} 13-\mathrm{C} 14$ & $1.390(7)$ \\
\hline $\mathrm{C} 3-\mathrm{C} 4$ & $1.363(7)$ & $\mathrm{C} 13-\mathrm{H} 13$ & 0.9300 \\
\hline $\mathrm{C} 3-\mathrm{H} 3 \mathrm{~A}$ & 0.9300 & $\mathrm{C} 14-\mathrm{C} 15$ & $1.380(7)$ \\
\hline $\mathrm{C} 4-\mathrm{C} 5$ & $1.392(7)$ & $\mathrm{C} 14-\mathrm{H} 14$ & 0.9300 \\
\hline $\mathrm{C} 4-\mathrm{H} 4$ & 0.9300 & $\mathrm{C} 15-\mathrm{N} 4$ & $1.332(6)$ \\
\hline $\mathrm{C} 5-\mathrm{C} 6$ & $1.369(6)$ & $\mathrm{C} 15-\mathrm{H} 15$ & 0.9300 \\
\hline $\mathrm{C} 5-\mathrm{H} 5$ & 0.9300 & $\mathrm{C} 16-\mathrm{N} 4$ & $1.343(5)$ \\
\hline $\mathrm{C} 6-\mathrm{C} 7$ & $1.364(6)$ & $\mathrm{C} 16-\mathrm{H} 16$ & 0.9300 \\
\hline $\mathrm{C} 6-\mathrm{C} 8$ & $1.512(6)$ & $\mathrm{N} 1-\mathrm{Co} 1$ & $2.093(4)$ \\
\hline $\mathrm{C} 7-\mathrm{N} 1$ & $1.339(5)$ & $\mathrm{N} 2-\mathrm{H} 2$ & 0.8600 \\
\hline $\mathrm{C} 7-\mathrm{H} 7$ & 0.9300 & $\mathrm{~N} 3-\mathrm{H} 3$ & 0.8600 \\
\hline $\mathrm{C} 8-\mathrm{N} 2$ & $1.454(5)$ & $\mathrm{N} 4-\mathrm{Co} 1^{\mathrm{iii}}$ & $2.155(4)$ \\
\hline $\mathrm{C} 8-\mathrm{H} 8 \mathrm{~A}$ & 0.9700 & $\mathrm{O} 1-\mathrm{Co} 1$ & $2.070(3)$ \\
\hline $\mathrm{C} 8-\mathrm{H} 8 \mathrm{~B}$ & 0.9700 & $\mathrm{O} 2-\mathrm{Co}^{\mathrm{ii}}$ & $2.117(3)$ \\
\hline $\mathrm{C} 9-\mathrm{O} 5$ & $1.215(5)$ & $\mathrm{O} 3-\mathrm{Co} 1$ & $2.072(3)$ \\
\hline $\mathrm{C} 9-\mathrm{N} 2$ & $1.311(5)$ & $\mathrm{O} 4-\mathrm{Co}^{\mathrm{i}}$ & $2.124(3)$ \\
\hline $\mathrm{C} 9-\mathrm{C} 10$ & $1.535(6)$ & $\mathrm{Co} 1-\mathrm{O} 2^{\mathrm{ii}}$ & $2.117(3)$ \\
\hline $\mathrm{C} 10-\mathrm{O} 6$ & $1.222(5)$ & $\mathrm{Co} 1-\mathrm{O} 4^{\mathrm{i}}$ & $2.124(3)$ \\
\hline $\mathrm{C} 10-\mathrm{N} 3$ & $1.322(5)$ & $\mathrm{Co} 1-\mathrm{N} 4^{\mathrm{iv}}$ & $2.155(4)$ \\
\hline $\mathrm{O} 4-\mathrm{C} 1-\mathrm{O} 3$ & $125.7(4)$ & $\mathrm{C} 12-\mathrm{C} 13-\mathrm{H} 13$ & 120.0 \\
\hline $\mathrm{O} 4-\mathrm{C} 1-\mathrm{C}^{\mathrm{i}}$ & $117.5(5)$ & $\mathrm{C} 14-\mathrm{C} 13-\mathrm{H} 13$ & 120.0 \\
\hline $\mathrm{O} 3-\mathrm{C} 1-\mathrm{C}^{\mathrm{i}}$ & $116.8(6)$ & $\mathrm{C} 15-\mathrm{C} 14-\mathrm{C} 13$ & $117.9(5)$ \\
\hline $\mathrm{O} 2-\mathrm{C} 2-\mathrm{O} 1$ & $125.5(4)$ & $\mathrm{C} 15-\mathrm{C} 14-\mathrm{H} 14$ & 121.0 \\
\hline $\mathrm{O} 2-\mathrm{C} 2-\mathrm{C} 2^{\mathrm{ii}}$ & $115.7(6)$ & $\mathrm{C} 13-\mathrm{C} 14-\mathrm{H} 14$ & 121.0 \\
\hline
\end{tabular}




\begin{tabular}{|c|c|c|c|}
\hline $\mathrm{O} 1-\mathrm{C} 2-\mathrm{C}^{\mathrm{ii}}$ & $118.8(5)$ & $\mathrm{N} 4-\mathrm{C} 15-\mathrm{C} 14$ & $123.5(5)$ \\
\hline $\mathrm{N} 1-\mathrm{C} 3-\mathrm{C} 4$ & $123.7(5)$ & $\mathrm{N} 4-\mathrm{C} 15-\mathrm{H} 15$ & 118.2 \\
\hline $\mathrm{N} 1-\mathrm{C} 3-\mathrm{H} 3 \mathrm{~A}$ & 118.2 & $\mathrm{C} 14-\mathrm{C} 15-\mathrm{H} 15$ & 118.2 \\
\hline $\mathrm{C} 4-\mathrm{C} 3-\mathrm{H} 3 \mathrm{~A}$ & 118.2 & $\mathrm{~N} 4-\mathrm{C} 16-\mathrm{C} 12$ & $124.1(4)$ \\
\hline $\mathrm{C} 3-\mathrm{C} 4-\mathrm{C} 5$ & $117.5(5)$ & $\mathrm{N} 4-\mathrm{C} 16-\mathrm{H} 16$ & 117.9 \\
\hline $\mathrm{C} 3-\mathrm{C} 4-\mathrm{H} 4$ & 121.2 & $\mathrm{C} 12-\mathrm{C} 16-\mathrm{H} 16$ & 117.9 \\
\hline $\mathrm{C} 5-\mathrm{C} 4-\mathrm{H} 4$ & 121.2 & $\mathrm{C} 7-\mathrm{N} 1-\mathrm{C} 3$ & $116.5(5)$ \\
\hline $\mathrm{C} 6-\mathrm{C} 5-\mathrm{C} 4$ & $120.4(5)$ & $\mathrm{C} 7-\mathrm{N} 1-\mathrm{Co} 1$ & $121.2(3)$ \\
\hline $\mathrm{C} 6-\mathrm{C} 5-\mathrm{H} 5$ & 119.8 & $\mathrm{C} 3-\mathrm{N} 1-\mathrm{Co} 1$ & $122.2(3)$ \\
\hline $\mathrm{C} 4-\mathrm{C} 5-\mathrm{H} 5$ & 119.8 & $\mathrm{C} 9-\mathrm{N} 2-\mathrm{C} 8$ & $120.0(4)$ \\
\hline $\mathrm{C} 7-\mathrm{C} 6-\mathrm{C} 5$ & $117.2(4)$ & $\mathrm{C} 9-\mathrm{N} 2-\mathrm{H} 2$ & 120.0 \\
\hline $\mathrm{C} 7-\mathrm{C} 6-\mathrm{C} 8$ & $121.4(4)$ & $\mathrm{C} 8-\mathrm{N} 2-\mathrm{H} 2$ & 120.0 \\
\hline $\mathrm{C} 5-\mathrm{C} 6-\mathrm{C} 8$ & $121.5(4)$ & $\mathrm{C} 10-\mathrm{N} 3-\mathrm{C} 11$ & $123.5(4)$ \\
\hline $\mathrm{N} 1-\mathrm{C} 7-\mathrm{C} 6$ & $124.7(5)$ & $\mathrm{C} 10-\mathrm{N} 3-\mathrm{H} 3$ & 118.3 \\
\hline $\mathrm{N} 1-\mathrm{C} 7-\mathrm{H} 7$ & 117.7 & $\mathrm{C} 11-\mathrm{N} 3-\mathrm{H} 3$ & 118.3 \\
\hline $\mathrm{C} 6-\mathrm{C} 7-\mathrm{H} 7$ & 117.7 & $\mathrm{C} 15-\mathrm{N} 4-\mathrm{C} 16$ & $116.4(4)$ \\
\hline $\mathrm{N} 2-\mathrm{C} 8-\mathrm{C} 6$ & $113.1(3)$ & $\mathrm{C} 15-\mathrm{N} 4-\mathrm{Co} 1^{\mathrm{iii}}$ & $122.3(3)$ \\
\hline $\mathrm{N} 2-\mathrm{C} 8-\mathrm{H} 8 \mathrm{~A}$ & 109.0 & $\mathrm{C} 16-\mathrm{N} 4-\mathrm{Co} 1^{\mathrm{iii}}$ & $120.8(3)$ \\
\hline $\mathrm{C} 6-\mathrm{C} 8-\mathrm{H} 8 \mathrm{~A}$ & 109.0 & $\mathrm{C} 2-\mathrm{O} 1-\mathrm{Co} 1$ & $112.5(3)$ \\
\hline $\mathrm{N} 2-\mathrm{C} 8-\mathrm{H} 8 \mathrm{~B}$ & 109.0 & $\mathrm{C} 2-\mathrm{O} 2-\mathrm{Co}{ }^{\mathrm{ii}}$ & $112.7(3)$ \\
\hline $\mathrm{C} 6-\mathrm{C} 8-\mathrm{H} 8 \mathrm{~B}$ & 109.0 & $\mathrm{C} 1-\mathrm{O} 3-\mathrm{Co} 1$ & $111.1(3)$ \\
\hline $\mathrm{H} 8 \mathrm{~A}-\mathrm{C} 8-\mathrm{H} 8 \mathrm{~B}$ & 107.8 & $\mathrm{C} 1-\mathrm{O} 4-\mathrm{Co}^{\mathrm{i}}$ & $110.2(3)$ \\
\hline $\mathrm{O} 5-\mathrm{C} 9-\mathrm{N} 2$ & $123.9(4)$ & $\mathrm{O} 1-\mathrm{Co} 1-\mathrm{O} 3$ & $163.58(13)$ \\
\hline $\mathrm{O} 5-\mathrm{C} 9-\mathrm{C} 10$ & $120.3(4)$ & $\mathrm{O} 1-\mathrm{Co} 1-\mathrm{N} 1$ & $95.51(15)$ \\
\hline $\mathrm{N} 2-\mathrm{C} 9-\mathrm{C} 10$ & $115.8(4)$ & $\mathrm{O} 3-\mathrm{Co} 1-\mathrm{N} 1$ & $99.50(14)$ \\
\hline $\mathrm{O} 6-\mathrm{C} 10-\mathrm{N} 3$ & $125.5(4)$ & $\mathrm{O} 1-\mathrm{Co} 1-\mathrm{O} 2^{\mathrm{ii}}$ & $79.59(13)$ \\
\hline $\mathrm{O} 6-\mathrm{C} 10-\mathrm{C} 9$ & $121.8(4)$ & $\mathrm{O} 3-\mathrm{Co} 1-\mathrm{O} 2^{\mathrm{ii}}$ & $84.77(12)$ \\
\hline $\mathrm{N} 3-\mathrm{C} 10-\mathrm{C} 9$ & $112.7(4)$ & $\mathrm{N} 1-\mathrm{Co} 1-\mathrm{O} 2^{\mathrm{ii}}$ & $172.33(14)$ \\
\hline $\mathrm{N} 3-\mathrm{C} 11-\mathrm{C} 12$ & $114.8(4)$ & $\mathrm{O} 1-\mathrm{Co} 1-\mathrm{O} 4^{\mathrm{i}}$ & $92.68(12)$ \\
\hline $\mathrm{N} 3-\mathrm{C} 11-\mathrm{H} 11 \mathrm{~A}$ & 108.6 & $\mathrm{O} 3-\mathrm{Co} 1-\mathrm{O} 4^{\mathrm{i}}$ & $80.86(12)$ \\
\hline $\mathrm{C} 12-\mathrm{C} 11-\mathrm{H} 11 \mathrm{~A}$ & 108.6 & $\mathrm{~N} 1-\mathrm{Co} 1-\mathrm{O} 4^{\mathrm{i}}$ & $89.62(14)$ \\
\hline $\mathrm{N} 3-\mathrm{C} 11-\mathrm{H} 11 \mathrm{~B}$ & 108.6 & $\mathrm{O} 2^{\mathrm{ii}}-\mathrm{Co} 1-\mathrm{O} 4^{\mathrm{i}}$ & $84.76(13)$ \\
\hline $\mathrm{C} 12-\mathrm{C} 11-\mathrm{H} 11 \mathrm{~B}$ & 108.6 & $\mathrm{O} 1-\mathrm{Co} 1-\mathrm{N} 4^{\mathrm{iv}}$ & $92.74(13)$ \\
\hline $\mathrm{H} 11 \mathrm{~A}-\mathrm{C} 11-\mathrm{H} 11 \mathrm{~B}$ & 107.6 & $\mathrm{O} 3-\mathrm{Co} 1-\mathrm{N} 4^{\mathrm{iv}}$ & $92.70(13)$ \\
\hline $\mathrm{C} 13-\mathrm{C} 12-\mathrm{C} 16$ & $117.9(4)$ & $\mathrm{N} 1-\mathrm{Co} 1-\mathrm{N} 4^{\mathrm{iv}}$ & $94.43(15)$ \\
\hline $\mathrm{C} 13-\mathrm{C} 12-\mathrm{C} 11$ & $125.3(4)$ & $\mathrm{O} 2^{\mathrm{ii}}-\mathrm{Co} 1-\mathrm{N} 4^{\mathrm{iv}}$ & $91.71(14)$ \\
\hline $\mathrm{C} 16-\mathrm{C} 12-\mathrm{C} 11$ & $116.8(4)$ & $\mathrm{O} 4-\mathrm{Co} 1-\mathrm{N} 4^{\mathrm{iv}}$ & $172.90(15)$ \\
\hline $\mathrm{C} 12-\mathrm{C} 13-\mathrm{C} 14$ & $120.0(5)$ & & \\
\hline $\mathrm{N} 1-\mathrm{C} 3-\mathrm{C} 4-\mathrm{C} 5$ & $0.2(9)$ & $\mathrm{C} 14-\mathrm{C} 15-\mathrm{N} 4-\mathrm{Co}^{1 \mathrm{ii}}$ & $171.3(5)$ \\
\hline $\mathrm{C} 3-\mathrm{C} 4-\mathrm{C} 5-\mathrm{C} 6$ & $1.7(8)$ & $\mathrm{C} 12-\mathrm{C} 16-\mathrm{N} 4-\mathrm{C} 15$ & $1.5(8)$ \\
\hline $\mathrm{C} 4-\mathrm{C} 5-\mathrm{C} 6-\mathrm{C} 7$ & $-2.3(7)$ & $\mathrm{C} 12-\mathrm{C} 16-\mathrm{N} 4-\mathrm{Co}{ }^{\mathrm{iii}}$ & $-171.1(4)$ \\
\hline $\mathrm{C} 4-\mathrm{C} 5-\mathrm{C} 6-\mathrm{C} 8$ & $177.0(5)$ & $\mathrm{O} 2-\mathrm{C} 2-\mathrm{O} 1-\mathrm{Co} 1$ & $-173.9(3)$ \\
\hline $\mathrm{C} 5-\mathrm{C} 6-\mathrm{C} 7-\mathrm{N} 1$ & $1.0(7)$ & $\mathrm{C} 22^{\mathrm{ii}}-\mathrm{C} 2-\mathrm{O} 1-\mathrm{Co} 1$ & $5.5(6)$ \\
\hline $\mathrm{C} 8-\mathrm{C} 6-\mathrm{C} 7-\mathrm{N} 1$ & $-178.3(4)$ & $\mathrm{O} 1-\mathrm{C} 2-\mathrm{O} 2-\mathrm{Co}^{\mathrm{ii}}$ & $-174.5(3)$ \\
\hline $\mathrm{C} 7-\mathrm{C} 6-\mathrm{C} 8-\mathrm{N} 2$ & $-121.0(5)$ & $\mathrm{C} 2{ }^{\mathrm{ii}}-\mathrm{C} 2-\mathrm{O} 2-\mathrm{Co}^{\mathrm{ii}}$ & $6.2(6)$ \\
\hline $\mathrm{C} 5-\mathrm{C} 6-\mathrm{C} 8-\mathrm{N} 2$ & $59.8(6)$ & $\mathrm{O} 4-\mathrm{C} 1-\mathrm{O} 3-\mathrm{Co} 1$ & $-166.2(4)$ \\
\hline $\mathrm{O} 5-\mathrm{C} 9-\mathrm{C} 10-\mathrm{O} 6$ & $174.2(4)$ & $\mathrm{C} 11^{\mathrm{i}}-\mathrm{C} 1-\mathrm{O} 3-\mathrm{Co} 1$ & $13.8(6)$ \\
\hline
\end{tabular}




$\begin{array}{llll}\mathrm{N} 2-\mathrm{C} 9-\mathrm{C} 10-\mathrm{O} 6 & -6.6(6) & \mathrm{O} 3-\mathrm{C} 1-\mathrm{O} 4-\mathrm{Co} 1^{\mathrm{i}} & -166.7(4) \\ \mathrm{O} 5-\mathrm{C} 9-\mathrm{C} 10-\mathrm{N} 3 & -6.6(6) & \mathrm{C} 1-\mathrm{C} 1-\mathrm{O} 4-\mathrm{Co} 1^{\mathrm{i}} & 13.4(6) \\ \mathrm{N} 2-\mathrm{C} 9-\mathrm{C} 10-\mathrm{N} 3 & 172.6(4) & \mathrm{C} 2-\mathrm{O} 1-\mathrm{Co}-\mathrm{O} 3 & 11.5(6) \\ \mathrm{N} 3-\mathrm{C} 11-\mathrm{C} 12-\mathrm{C} 13 & -6.9(7) & \mathrm{C} 2-\mathrm{O} 1-\mathrm{Co} 1-\mathrm{N} 1 & 167.5(3) \\ \mathrm{N} 3-\mathrm{C} 11-\mathrm{C} 12-\mathrm{C} 16 & 174.7(4) & \mathrm{C} 2-\mathrm{O} 1-\mathrm{Co} 1-\mathrm{O} 2^{\mathrm{ii}} & -6.5(3) \\ \mathrm{C} 16-\mathrm{C} 12-\mathrm{C} 13-\mathrm{C} 14 & -1.3(9) & \mathrm{C} 2-\mathrm{O} 1-\mathrm{Co} 1-\mathrm{O} 4^{\mathrm{i}} & 77.7(3) \\ \mathrm{C} 11-\mathrm{C} 12-\mathrm{C} 13-\mathrm{C} 14 & -179.7(5) & \mathrm{C} 2-\mathrm{O} 1-\mathrm{Co} 1-\mathrm{N} 4^{\mathrm{iv}} & -97.7(3) \\ \mathrm{C} 12-\mathrm{C} 13-\mathrm{C} 14-\mathrm{C} 15 & 1.6(10) & \mathrm{C} 1-\mathrm{O} 3-\mathrm{Co} 1-\mathrm{O} 1 & 51.8(6) \\ \mathrm{C} 13-\mathrm{C} 14-\mathrm{C} 15-\mathrm{N} 4 & -0.3(10) & \mathrm{C} 1-\mathrm{O} 3-\mathrm{Co} 1-\mathrm{N} 1 & -104.0(3) \\ \mathrm{C} 13-\mathrm{C} 12-\mathrm{C} 16-\mathrm{N} 4 & -0.3(8) & \mathrm{C} 1-\mathrm{O} 3-\mathrm{Co} 1-\mathrm{O} 2^{\mathrm{ii}} & 69.6(3) \\ \mathrm{C} 11-\mathrm{C} 12-\mathrm{C} 16-\mathrm{N} 4 & 178.2(4) & \mathrm{C} 1-\mathrm{O} 3-\mathrm{Co} 1-\mathrm{O} 4^{\mathrm{i}} & -15.9(3) \\ \mathrm{C} 6-\mathrm{C} 7-\mathrm{N} 1-\mathrm{C} 3 & 0.9(7) & \mathrm{C} 1-\mathrm{O} 3-\mathrm{Co} 1-\mathrm{N} 4^{\mathrm{iv}} & 161.1(3) \\ \mathrm{C} 6-\mathrm{C} 7-\mathrm{N} 1-\mathrm{Co} 1 & 178.1(3) & \mathrm{C} 7-\mathrm{N} 1-\mathrm{Co} 1-\mathrm{O} 1 & -142.6(3) \\ \mathrm{C} 4-\mathrm{C} 3-\mathrm{N} 1-\mathrm{C} 7 & \mathrm{C} 3-\mathrm{N} 1-\mathrm{Co} 1-\mathrm{O} 1 & 34.4(4) \\ \mathrm{C} 4-\mathrm{C} 3-\mathrm{N} 1-\mathrm{Co} 1 & -1.5(8) & \mathrm{C} 7-\mathrm{N} 1-\mathrm{Co} 1-\mathrm{O} 3 & 30.7(4) \\ \mathrm{O} 5-\mathrm{C} 9-\mathrm{N} 2-\mathrm{C} 8 & -178.7(4) & \mathrm{C} 3-\mathrm{N} 1-\mathrm{Co} 1-\mathrm{O} 3 & -152.3(4) \\ \mathrm{C} 10-\mathrm{C} 9-\mathrm{N} 2-\mathrm{C} 8 & 0.8(7) & \mathrm{C} 7-\mathrm{N} 1-\mathrm{Co} 1-\mathrm{O} 2^{\mathrm{ii}} & -92.7(11) \\ \mathrm{C} 6-\mathrm{C} 8-\mathrm{N} 2-\mathrm{C} 9 & -178.3(4) & \mathrm{C} 3-\mathrm{N} 1-\mathrm{Co} 1-\mathrm{O} 2^{\mathrm{ii}} & 84.3(11) \\ \mathrm{O} 6-\mathrm{C} 10-\mathrm{N} 3-\mathrm{C} 11 & 64.2(6) & \mathrm{C} 7-\mathrm{N} 1-\mathrm{Co} 1-\mathrm{O} 4^{\mathrm{i}} & -50.0(4) \\ \mathrm{C} 9-\mathrm{C} 10-\mathrm{N} 3-\mathrm{C} 11 & 1.6(7) & \mathrm{C} 3-\mathrm{N} 1-\mathrm{Co} 1-\mathrm{O} 4^{\mathrm{i}} & 127.0(4) \\ \mathrm{C} 12-\mathrm{C} 11-\mathrm{N} 3-\mathrm{C} 10 & -177.5(4) & \mathrm{C} 7-\mathrm{N} 1-\mathrm{Co} 1-\mathrm{N} 4^{\mathrm{iv}} & 124.2(4) \\ \mathrm{C} 14-\mathrm{C} 15-\mathrm{N} 4-\mathrm{C} 16 & -77.4(6) & \mathrm{C} 3-\mathrm{N} 1-\mathrm{Co} 1-\mathrm{N} 4^{\mathrm{iv}} & -58.8(4) \\ & -1.2(9) & & \end{array}$

Symmetry codes: (i) $-x+1,-y,-z+1$; (ii) $-x,-y,-z+1$; (iii) $x+1,-y+1 / 2, z-1 / 2$; (iv) $x-1,-y+1 / 2, z+1 / 2$.

Hydrogen-bond geometry $\left(\AA,{ }^{\circ}\right)$

\begin{tabular}{lllll}
\hline$D-\mathrm{H}^{\cdots} \cdots A$ & $D-\mathrm{H}$ & $\mathrm{H} \cdots A$ & $D \cdots A$ & $D-\mathrm{H} \cdots A$ \\
\hline $\mathrm{N} 3-\mathrm{H} 3 \cdots \mathrm{O}^{\mathrm{v}}$ & 0.86 & 2.14 & $2.863(5)$ & 142 \\
\hline
\end{tabular}

Symmetry code: (v) $x,-y+1 / 2, z+1 / 2$. 